\title{
Tonsillectomy with Adenoidectomy
}

National Cancer Institute

\section{Source}

National Cancer Institute. Tonsillectomy with Adenoidectomy. NCI Thesaurus. Code C51684.

Surgical removal of the tonsils and adenoids. 